\title{
Some New Aspects of the Joint Effect of Rotation and Topography on Internal Solitary Waves
}

\author{
LEV A. OSTROVSKY \\ University of Colorado Boulder, Boulder, Colorado, and University of North Carolina at \\ Chapel Hill, Chapel Hill, North Carolina \\ KARL R. HELFRICH \\ Department of Physical Oceanography, Woods Hole Oceanographic Institution, \\ Woods Hole, Massachusetts
}

(Manuscript received 1 August 2018, in final form 5 April 2019)

\begin{abstract}
Using a recently developed asymptotic theory of internal solitary wave propagation over a sloping bottom in a rotating ocean, some new qualitative and quantitative features of this process are analyzed for internal waves in a two-layer ocean. The interplay between different singularities-terminal damping due to radiation and disappearing quadratic nonlinearity, and reaching an "internal beach" (e.g., zero lower-layer depth)-is discussed. Examples of the adiabatic evolution of a single solitary wave over a uniformly sloping bottom under realistic conditions are considered in more detail and compared with numerical solutions of the variablecoefficient, rotation-modified Korteweg-de Vries (rKdV) equation.
\end{abstract}

\section{Introduction}

The experiments of the last decade have confirmed that Earth's rotation can significantly affect the longrange propagation of nonlinear mesoscale internal waves in the ocean (e.g., Farmer et al. 2009; Grimshaw et al. 2017). It is also known that rotation destroys internal solitons due to a resonant radiation of inertia-gravity waves (terminal damping; see Grimshaw et al. 1998a). (Note that a soliton, in the formal mathematical sense, cannot exist in the presence of either rotation or inhomogeneity. However, here we use that term interchangeable with solitary wave.) Since the real oceanic conditions include horizontally inhomogeneous environments (e.g., varying bottom topography), there exists an interplay of several effects: terminal damping due to radiation, change in sign of the quadratic nonlinear term (e.g., the point of equal layer depths in a two-layer stratification), as well as a large amplitude increase, and possible breaking, when approaching the point where the pycnocline intersects the bottom. Grimshaw et al. (2014) studied these effects in a rotating medium using the rotation-modified

Corresponding author: Lev A. Ostrovsky, lev.ostrovsky@ gmail.com
Korteweg-de Vries (“Ostrovsky" or rKdV equation) for nonlinear waves in rotating ocean (Ostrovsky 1978). Recently, Yuan et al. (2018) applied a similar equation to undular bores. As part of their study, Grimshaw et al. (2014) derived from the rKdV model an adiabatic theory for the amplitude evolution of a soliton under the joint effects of variable depth and rotation. The specific calculations in Grimshaw et al. (2014) refer to numerical solutions of the full $\mathrm{rKdV}$ model applied to wave propagation in the South China Sea, without a comparison with the adiabatic theory.

This paper discusses some interesting properties of adiabatic soliton dynamics in a rotating fluid over a variable bottom with a focus on internal solitary waves in a two-layered ocean and a constant bottom slope. Whereas in a horizontally homogeneous layer a soliton adiabatically decreases to zero at a finite distance so that all its energy transforms to radiation (terminal damping), in a horizontally inhomogeneous environment the scenarios of a soliton propagating toward the beach can be qualitatively different. In general, there exist several possible scenarios of soliton decay. One possibility is still terminal damping described above. Another possibility is that a soliton reaches the beach before full radiation damping occurs, and the adiabatic formula works until 
the soliton amplitude increases as a beach is approached such that the nonlinearity becomes strong enough to make the basic, weakly nonlinear $\mathrm{rKdV}$ equation invalid. In this case the expected result is wave breaking near the coast. For an internal wave there is a third possibility: the wave reaches the point where the coefficient of the nonlinear term in the $\mathrm{rKdV}$ equation equals zero and in the adiabatic theory the solitary wave amplitude also goes to zero. This effect is possible for general stratifications and, in particular, at the location of equal layer depths in a two-layer stratification. These scenarios are discussed below for oceanographically relevant parameters and then the adiabatic predictions of soliton amplitude are compared to full numerical solutions of the variable-coefficient $\mathrm{rKdV}$ model. The case of offshore propagation of solitary waves is also considered. Of particular interest, regardless of propagation direction, is the competition between variable depth and radiation damping effects.

\section{Basic equations}

We consider the rKdV equation modified by a sloping bottom in the notations used in Grimshaw et al. (2014):

$\frac{\partial}{\partial x}\left(\frac{\partial \eta}{\partial t}+c \frac{\partial \eta}{\partial x}+\mu \eta \frac{\partial \eta}{\partial x}+\lambda \frac{\partial^{3} \eta}{\partial x^{3}}+\frac{c}{2} \frac{Q_{x}}{Q} \eta\right)=\gamma \eta$,

where $\eta(x, t)$ is the maximum vertical displacement of a wave mode. Here $c(x)$ is the linear long wave phase speed obtained from the solution of the standard eigenvalue problem for the vertical structure function $\phi(z)$, which is itself a slowly varying function of $x$. The other slowly varying coefficients $\mu(x), \lambda(x), \gamma(x)$, and $Q(x)$ can be expressed as integrals of $\phi$ or $\phi^{\prime}$ over the water depth $h(x)$. Their general expressions for internal waves are given in many publications (e.g., Grimshaw et al. 2014). In the absence of a mean flow, $\gamma=f^{2} / 2 c$, where $f$ is the Coriolis frequency. The parameter $Q$ has been discussed in numerous publications beginning in the 1970s (Pelinovsky et al. 1977). For long internal waves in a Boussinesq fluid in the absence of a mean background current

$$
Q=2 c^{3} \int_{-h(x)}^{0}\left(\frac{d \phi}{d z}\right)^{2} d z
$$

Frequently $Q$ is normalized with a constant, say the value of $Q$ at $x=0$; however, it is clear from (1) that this normalization, or any constant factor, is not important. However, when (2) is used, (1/2) $Q \eta^{2}$ is formally the version of the wave action flux whose integral in time $t$ gives the wave energy (per unit density).
Adiabatic solution for a slowly varying $\mathrm{KdV}$ soliton governed by (1) was obtained in Grimshaw et al. (2014). The soliton, with slowly varying parameters, can be written in the form

$$
\eta=a Q^{-1 / 2} \operatorname{sech}^{2}(K \theta),
$$

where

$$
\theta=-t+\int_{0}^{x} \frac{d x}{c}-\int_{0}^{x} \frac{V}{c} d x, \quad V=\frac{\mu a}{3 Q^{1 / 2}}=\frac{4 \lambda}{c^{2}} K^{2}
$$

(here the integrals in the phase $\theta$ are written in terms of $x$ ). Using standard asymptotic procedures it was shown that the amplitude function $a(x)$ varies as (we use a slightly different but equivalent form of the solution):

$$
\begin{aligned}
\frac{a(x)}{a_{0}} & =\frac{w_{0}}{w}\left[1-\left(\frac{w_{0}^{3}}{a_{0}}\right)^{1 / 2} \int_{0}^{x} \gamma\left(\frac{w}{w_{0}}\right)^{2} d x^{\prime}\right]^{2}, \\
w & =\left(\frac{12 \lambda}{\mu c^{2}} Q^{1 / 2}\right)^{1 / 3} .
\end{aligned}
$$

The subscript 0 refers to the position at $x=0$.

For a homogeneous rotating medium $c, \mu, \gamma, \lambda$, and $Q$ are constants and (5) reduces to the well-known formula for the terminal decay of $\mathrm{KdV}$ soliton with rotation (Grimshaw et al. 1998a):

$$
A=A_{0}\left(1-\frac{x}{X_{e}}\right)^{2}, \quad X_{e}=\frac{c}{\gamma}\left(\frac{\mu A_{0}}{12 \lambda}\right)^{1 / 2} .
$$

Here and further $A=a Q^{-1 / 2}$ is the soliton amplitude. According to (6) a soliton completely decays by radiation to a trailing inertia-gravity wave train at a finite distance $X_{e}$. The trailing radiation will eventually organize into one or more nonlinear wave packets, or envelope solitons (Helfrich 2007; Grimshaw and Helfrich 2008; Whitfield and Johnson 2014).

The general adiabatic solution (5) already implies the potential existence of singularities mentioned above. One of them occurs on the beach. In general, since the conserved soliton energy is distributed over a smaller depth $h$ as the wave propagated onshore, its amplitude in terms of the displacement and/or horizontal fluid velocity, as well as its steepness should infinitely increase when the water depth $h$ goes to zero, so that the nonlinearity becomes strong and the weakly nonlinear description is not applicable anymore. A typical result is breaking of the wave near the beach. However, the wave may not reach the beach as a soliton if either the rotational term in the brackets goes to zero or $w \rightarrow 0$. The latter happens when the coefficient of the nonlinear term $\mu=0$. In either case, formally the soliton amplitude goes 
to zero. Certainly, the total energy is conserved, and the destruction of a soliton turns the energy to a nonstationary wave packet that continues moving onshore, but the soliton as a localized entity disappears, and the above adiabatic formulas evidently fail beyond this location.

\section{Internal waves in a two-layer fluid}

Given a general background stratification and bottom topography, the rKdV coefficients could be obtained as functions of $x$ and (5) could be integrated numerically to determine the soliton amplitude $A(x)$. However, to get physical insight it is instructive to consider a Boussinesq, two-layer, rigid-lid model of the ocean with a constant upper-layer thickness $h_{1}$ and a varying lower-layer thickness $h_{2}(x)$. In this case the parameters of the $\mathrm{rKdV}$ model are

$$
\begin{aligned}
& c=\left(g^{\prime} \frac{h_{1} h_{2}}{h_{1}+h_{2}}\right)^{1 / 2}, \quad \mu=\frac{3}{2} c \frac{h_{1}-h_{2}}{h_{1} h_{2}}, \\
& \lambda=\frac{c}{6} h_{1} h_{2}, \quad Q=2 g^{\prime} c .
\end{aligned}
$$

Here $g^{\prime}=g \Delta \rho / \rho_{1}$ is the reduced gravity, $\Delta \rho=\rho_{2}-\rho_{1}$ is the density difference between the lower and upper layers, and $g$ is the gravitational acceleration. While the relation for $Q$ can be found directly from (2), it can be shown more generally that for the two-layer model it is proportional to $c(x)$ (see the appendix), which can significantly simplify calculations. Defining $s(x)=h_{2}(x) / h_{1}$ and using (7), the adiabatic solution (5) can now be written in the form

$$
\begin{aligned}
\frac{A}{A_{0}}= & {\left[\frac{s_{0}^{2}(s-1)}{s^{2}\left(s_{0}-1\right)}\right]^{1 / 3}\left\{1-q_{1} \int_{s_{0}}^{s}\left(\frac{1+s}{s}\right)\right.} \\
& \left.\times\left[\frac{s^{2}\left(s_{0}-1\right)}{s_{0}^{2}(s-1)}\right]^{2 / 3}\left(\frac{d x}{d s}\right) d s\right\}^{2},
\end{aligned}
$$

where $q_{1}=f^{2} h_{20} / g^{\prime} \sqrt{3 A_{0}\left(h_{1}-h_{20}\right)}$. Here $s_{0}=h_{20} / h_{1}$. Recall that for $s_{0}>1(<1)$ the initial solitary wave is a wave of depression (elevation) such that $A_{0}\left(1-s_{0}\right)>0$.

To obtain an analytical result we consider the bottom topography with a constant slope,

$$
h_{2}=h_{20}\left[1-\left(1-s_{0}^{-1}\right) \frac{x}{L}\right] \text {, }
$$

where $L$ is the distance from $x=0$ to where $s=1$. In this case (8) yields

$$
\frac{A}{A_{0}}=\left[\frac{s_{0}^{2}(s-1)}{s^{2}\left(s_{0}-1\right)}\right]^{1 / 3}(1-q I)^{2} .
$$

Here $q=q_{1} d x / d s=-f^{2} h_{20} L /\left(s_{0}-1\right) g^{\prime} \sqrt{3 A_{0}\left(h_{1}-h_{20}\right)}$ $<0$ and $I=I\left(s ; s_{0}\right)$ is the integral in (8) with the constant $d x / d s$ removed from the integrand. As shown below $I=I\left(1 ; s_{0}\right)<0$ for $s_{0}>1$ so that $q I>0$. It has the analytic form

$$
I=\frac{-3}{10(s-1)^{2 / 3} s_{0}^{4 / 3}}\left\{\begin{array}{l}
\left(s_{0}-1\right)^{2 / 3}\left(7 s^{4 / 3}+2 s^{7 / 3}-9 s^{1 / 3}\right)- \\
(s-1)^{2 / 3}\left(7 s_{0}^{4 / 3}+2 s_{0}^{7 / 3}-9 s_{0}^{1 / 3}\right)+ \\
9\left(s_{0}+s-s s_{0}-1\right)\left[s_{2}^{1 / 3} F_{1}\left(\frac{1}{3}, \frac{2}{3}, \frac{4}{3}, s\right)-s_{0}^{1 / 3} F_{1} F_{1}\left(\frac{1}{3}, \frac{2}{3}, \frac{4}{3}, s_{0}\right)\right.
\end{array}\right\} .
$$

Here ${ }_{2} F_{1}$ is the Gaussian hypergeometric function.

In the absence of rotation $(q=0)$ the soliton amplitude variation in (10) becomes

$$
\frac{A}{A_{0}}=\left[\frac{s_{0}^{2}(s-1)}{s^{2}\left(s_{0}-1\right)}\right]^{1 / 3} .
$$

As was shown by Talipova et al. (2015), in this case the ratio $A / A_{0}$ has a maximum at $s=2$ which, evidently, exists only if $s_{0}>2$. At $s=1$ the soliton amplitude goes to zero. This is location of equal layer depths where the nonlinear coefficient $\mu=0$, see (7). In this nonrotating case, if $s_{0}<1$ and the soliton propagates into deeper water then the amplitude decreases monotonically to zero as $s \rightarrow 1$ is approached from below. The same happens if $s_{0}>1$ and the soliton propagates into shallower water.

In the general case, the integral $I$ in (11) is real for $s \rightarrow 1$ and at a given $s_{0}$ it has a finite limit $I_{\text {lim }}\left(s_{0}\right)$. When $s_{0}>1$ and $s \rightarrow 1$ from above (propagation from deep to shallow water) $I_{\lim }\left(s_{0}\right)<0$ and its modulus increases with $s_{0}$ (Fig. 1). The product $q I \geq 0$. The modulus of $q$ in (10) decreases with the initial soliton amplitude $A_{0}$ and increases with the inhomogeneity scale $L$. As a result, two scenarios are possible. If $q I_{\text {lim }}<1$, the soliton amplitude goes to zero due to the first factor in (10), see (12). 


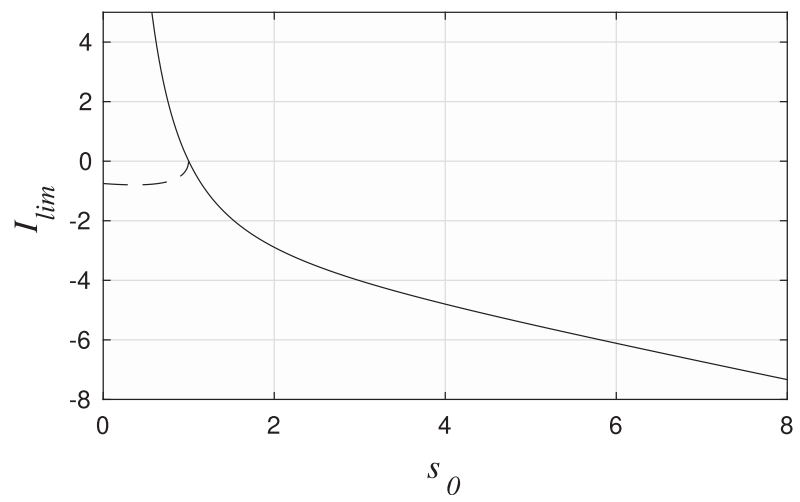

FIG. 1. Plot of $I_{\lim }\left(s_{0}\right)$. The solid line is for wave propagation from $s_{0}$ to $s \rightarrow 1$ (the point of polarity reversal), and the dashed line is for propagation toward an internal beach, $s \rightarrow 0$, for $s_{0}<1$.

In other words, the nonlinear term in the $\mathrm{rKdV}$ equation (1) becomes zero. If, however, $q I_{\text {lim }}>1$, then terminal damping occurs first, at some $s>1$, that is, before the point of equal depths. Below we consider both these cases.

Conversely, when propagation is from shallow to deep water with $s_{0}<1$, the critical point, $s \rightarrow 1$, is approached from below, and $I_{\lim }\left(s_{0}\right)>0$ (Fig. 1). The depth $h_{2}(x)$ is again given by (9), but now $d x / d s=L /\left(1-s_{0}\right)>0$, $q=q_{1} d x / d s=f^{2} h_{20} L /\left(1-s_{0}\right) g^{\prime} \sqrt{3 A_{0}\left(h_{1}-h_{20}\right)}>0$, and again $q I \geq 0$.

The focus here is on the two situations above in which extinction due to the approach to a point of polarity reversal competes with radiation decay, and especially the case of a wave of depression propagating onshore since it is the most commonly observed situation. However, two additional situations are possible. The first is onshore propagation, for $s_{0}<1$, of a solitary wave of elevation toward the "internal" beach, $s \rightarrow 0\left(h_{2} \rightarrow 0\right)$. Then amplitude magnification, up to breaking [see (12)], competes with extinction by radiation decay. The integral $I\left(s, s_{0}\right)$ in $(8)$ remains the same except that $I_{\lim }$ is now found by integration from $s_{0}$ to 0 and is negative (see Fig. 1). Taking $s=s_{0}(1-x / L)$, where now $L$ is the distance to where $s=0$, gives $d x / d s=-s_{0} / L<0$, so that $q I \geq 0$ and extinction due to radiation decay before reaching the beach is possible.

The last possibility is offshore propagation for $s_{0}>1$. This principally involves extinction due to radiation decay. In the absence of rotation, (12) shows that increasing lower-layer depth continuously decreases the wave amplitude as $A \sim s^{-1 / 3}$, but does not introduce a distinguished point of extinction. With rotation, eventually a point where $q I=1$ will be reached and the solitary wave will be extinguished.

\section{On the applicability of the adiabatic approximation}

Before considering physical examples it is constructive to estimate the parametric range in which the adiabatic formulae are applicable. Similar to the KdV case, the $\mathrm{rKdV}$ equation (1) is valid when the first two terms dominate but, as known, in the variables $\tau=\int d x / c-t$ and $\xi=x$, they reduce to the term $\eta_{\xi}$, which in general, at least in the case of the solitary solution (3), is of the same order as the following two terms. The other two terms describing inhomogeneity through $Q$ and rotation, can be of the same order. However, in the adiabatic development when the soliton (3) is assumed to exist as a localized entity for a sufficiently long time, both of those terms must be small compared to the standard $\mathrm{KdV}$ terms in (1). In particular, the smallness of the rotational term is characterized by the Ostrovsky number, $O_{S}$, first introduced by Farmer et al. (2009), later formalized as $O_{S}=3 \mu \gamma^{-1} \eta_{x x}$ in Grimshaw et al. (2012), and used by Grimshaw et al. (2014). For our purposes we define it directly as the ratio between the nonlinear and rotational terms in (1):

$$
O_{S}=\frac{\left(\mu \eta \eta_{x}\right)_{x}}{\gamma \eta} \sim \frac{2 c \mu A_{0}}{f^{2} \Delta^{2}},
$$

where the appropriate length scale, $\Delta=c / K=\left(12 \lambda / \mu A_{0}\right)^{1 / 2}$, is the characteristic soliton width. In the examples considered below the condition $O_{S} \gg 1$ is always met initially. In a homogeneous medium, decrease of the solitary wave amplitude due to rotation eventually makes $O_{S}$ of the order of unity which destroys the solitary wave as a distinguished entity and only an oscillatory radiated wave remains (the full domain integrated energy is still conserved).

In the presence of inhomogeneity the situation is more complex. The relative effect of radiation and inhomogeneity depends on the ratio of the terms with $Q$ and $\gamma$ in (1). This ratio (we call it "competition parameter") scales as

$G_{S} \approx \frac{c}{\gamma} \frac{Q_{x}}{Q} \frac{\eta_{x}}{\eta} \sim \frac{c}{\gamma L \Delta}=\frac{c}{\gamma L}\left(\frac{\mu A_{0}}{12 \lambda}\right)^{1 / 2}=\frac{X_{e}}{L}$,

where $Q$ varies on $L, \eta$ on $\Delta$, with $X_{e}$ from (6). For large $G_{S}$ rotational effects can be neglected in the adiabatic theory. Still, the condition of smallness of the term $Q$ term in (1), namely $\Delta \ll L$, should be met. In the opposite case, $G_{S} \ll 1$, the terminal damping occurs over a distance $\sim X_{e}$, much smaller than $L$ over which the environment is nearly homogeneous. From (14) rotational damping effects increase with increasing $f$ and decreasing $\left|A_{0}\right|$. 
One additional consideration regarding the applicability of the adiabatic solution (5) is that it is derived from the $\mathrm{rKdV}$ model (1) with quadratic nonlinearity. It is well known that as point where $\mu=0$ is approached the soliton evolves into a leading rarefaction followed by an undular bore and that beyond this point the undular bore may evolve into one or more solitary waves of reversed polarity followed by a dispersive wave train (Helfrich et al. 1984; Grimshaw et al. 1998b; Yuan et al. 2018). Modeling of this scattering process frequently involves addition of cubic nonlinearity (using the Gardner equation) which will be significant where $\mu \rightarrow 0$. However, the focus here is on the competition between spatial inhomogeneity and rotation in the approach to this point, where the cubic nonlinearity is less important.

\section{Examples}

\section{a. Onshore propagation for $s_{0}>1$}

To illustrate the competition between adiabatic internal soliton decay through inhomogeneity versus radiation damping consider a generic shelf area, two-layer stratification with $h_{1}=50 \mathrm{~m}, h_{20}=450 \mathrm{~m}$ (a total depth at $x=0$ of $\left.h_{0}=h_{1}+h_{20}=500 \mathrm{~m}\right), f=10^{-4} \mathrm{~s}^{-1}$, and $g^{\prime}=5 \times 10^{-3} \mathrm{~m} \mathrm{~s}^{-2}$. The initial solitary wave amplitude $A_{0}=-10 \mathrm{~m}$ and three topographic lengths $L=44.5$, 89 , and $178 \mathrm{~km}$ are considered with $h_{2}(x)$ from (9).

Figure 2 shows the adiabatic solution $A / A_{0}$ as a function of $s$ from (10) for these parameters (solid lines) with $L$ indicated. The nonrotating solution (12) is also shown (dashed line). Note that when plotted versus $s$, $A / A_{0}$ in (12) is independent of $L$, although the $x$ dependence is carried parametrically through $s(x)$ in (9). These parameters give $O_{S} \gg 1$ for all $L$ and $G_{S}=2.46$, 1.23 , and 0.62 for increasing $L$. Without rotation both $O_{S}$ and $G_{S}$ are infinite. The two shorter slopes result in complete decay of the soliton at $s=1$, although there are substantial effects of rotational decay prior to this location. The longer slope results in complete decay before the location where $h_{2}=h_{1}$. Setting $q I_{\text {lim }}=1$ shows that for rotational decay to occur before $s=1$ requires $L>122.9 \mathrm{~km}$ and $G_{S}<0.9$ for this particular set of parameters.

Numerical solutions of the inhomogeneous $\mathrm{rKdV}$ model (1) were used to test the adiabatic model (10) [and by extension the general version (5)]. The rKdV equation was first converted from the $(x, t)$ to the $(\tau, \xi)$ system and solved using a de-aliased, pseudospectral Fourier representation in $\tau$ and third-order RungeKutta integration in $\xi=x$. The solutions were initiated with a $\mathrm{KdV}$ soliton at $x=0$ and followed up the slope that transitioned to a constant depth shelf for $x \geq L\left(s_{0}-0.5\right) /\left(s_{0}-1\right)$, the location where $s=0.5$. Since

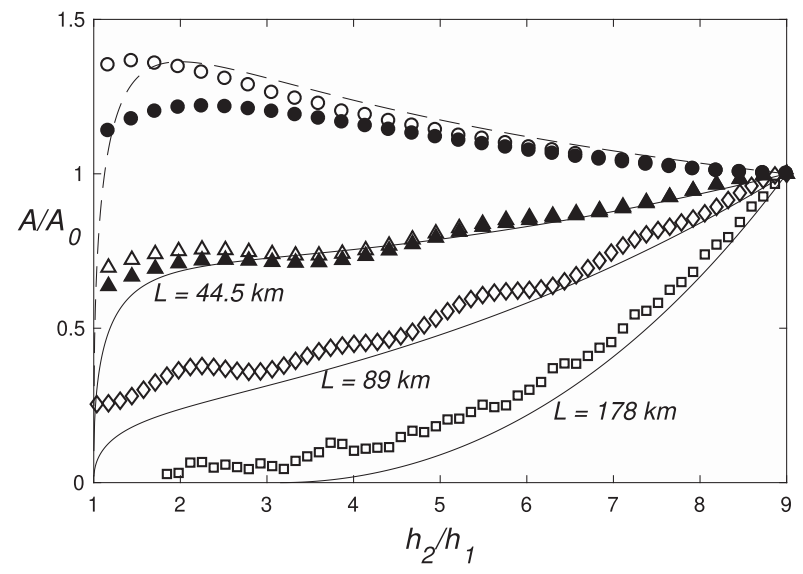

FIG. 2. Adiabatic theory (lines) vs KdV and rKdV numerical results (open symbols) for onshore propagation with $s_{0}=9 . A_{0}=-10 \mathrm{~m}$, $h_{1}=50 \mathrm{~m}, h_{20}=450 \mathrm{~m}, \Delta \rho / \rho_{1}=5.1 \times 10^{-4}, f=10^{-4} \mathrm{~s}^{-1}$ and the uniform slope topography with $L=44.5,89$, and $178 \mathrm{~km}$. The dashed line is for the nonrotating case (valid for all $L$ ), and the solid lines are for the rotating cases with $L$ as indicated. The open symbols are for the respective $\mathrm{KdV}$ (computed with $L=44.5 \mathrm{~km}$ ) and $\mathrm{rKdV}$ numerical solutions. The solid symbols are for companion calculations from the nonrotating and rotating Gardner equations for $L=44.5 \mathrm{~km}$.

the focus of this note is on the adiabatic theory in the approach to this point, the numerical solutions were not carried much beyond this transition.

Figure 3 shows the nonrotating $\mathrm{KdV}$ case computed with $L=44.5 \mathrm{~km}$. The solution $\eta$ is shown as a function of the shifted time $t-\int c^{-1} d x=-\tau$, with $t=0$ arbitrarily set to the arrival at $x=0$ of the peak of the initial solitary wave. These are effectively time series at $x$ locations indicated on the vertical axis. Note that in this figure and similar ones below amplitude and distance are nondimensionalized using $h_{0}=h_{1}+h_{20}$ and time by $\left(h_{0} / g^{\prime}\right)^{1 / 2}$. The solid dots indicate the peak amplitude of the leading disturbance (i.e., the soliton) at each $x$ used to compare with the adiabatic theory. These amplitudes are shown in Fig. 2 by the open circles. The agreement is quite good for $s>2$, but the KdV solution does not approach zero as $s \rightarrow 1$. This is not surprising since the adiabatic theory does not account for the evolution of the trailing wave train. Thus distinguishing the primary solitary wave from the rarefaction is ambiguous for $s<2$.

The same situation with rotation is shown in Fig. 4. The lead soliton amplitude is indicated with the open triangles in Fig. 2. Again the agreement with the adiabatic model is quite good. The small oscillations in soliton amplitude evident in Fig. 2 are the consequence of the periodic boundary conditions in $\tau$ that allows trailing inertia-gravity wave radiation to wrap around the domain. Note that only a portion of the $\tau$ domain is shown 

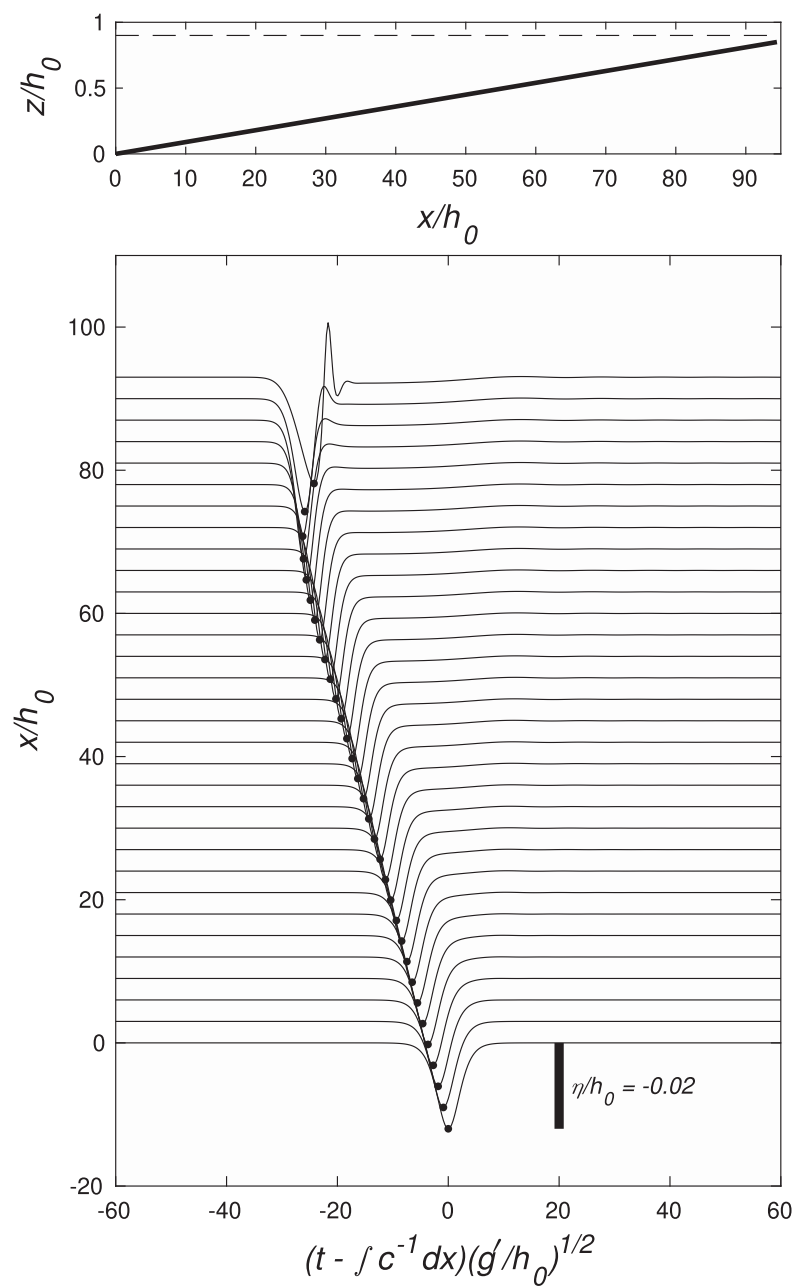

FIG. 3. Nonrotating KdV solution for the parameters of Fig. 2 and $L=44.5 \mathrm{~km}$. (top) The topography (heavy solid line) and the mean interface depth (dashed). (bottom) The numerical solution $\eta$ as a function of the shifted time $t-\int c^{-1} d x$, arbitrarily centered on the initial solitary wave. Here $x, z$, and $\eta$ are scaled with $h_{0}$ and $\tau$ with $\left(h_{0} / g^{\prime}\right)^{1 / 2}$. The $x$ locations of each time series of $\eta$ are indicated by the vertical axis. The dots show the local minima used to define the amplitude of the evolving solitary wave used in Fig. 2. Only a portion of the full $\tau$ domain is shown.

in these figures. The same difficulty distinguishing between the lead soliton and emerging rarefaction is present as $s \rightarrow 1$. It is worth noting that even for this relatively short propagation distance the effect of radiation damping on the soliton amplitude is significant and could be mistaken for dissipative losses (e.g., bottom friction) or wave instabilities in ocean observations.

Figure 5 shows the rKdV solution for the longest slope $L=178 \mathrm{~km}$. Here the initial soliton is completely extinguished by the radiation damping before the point $s=1$. The agreement with the adiabatic model in Fig. 2 (squares) is quite good with the amplitudes only slightly larger than those given by the adiabatic theory. (The same
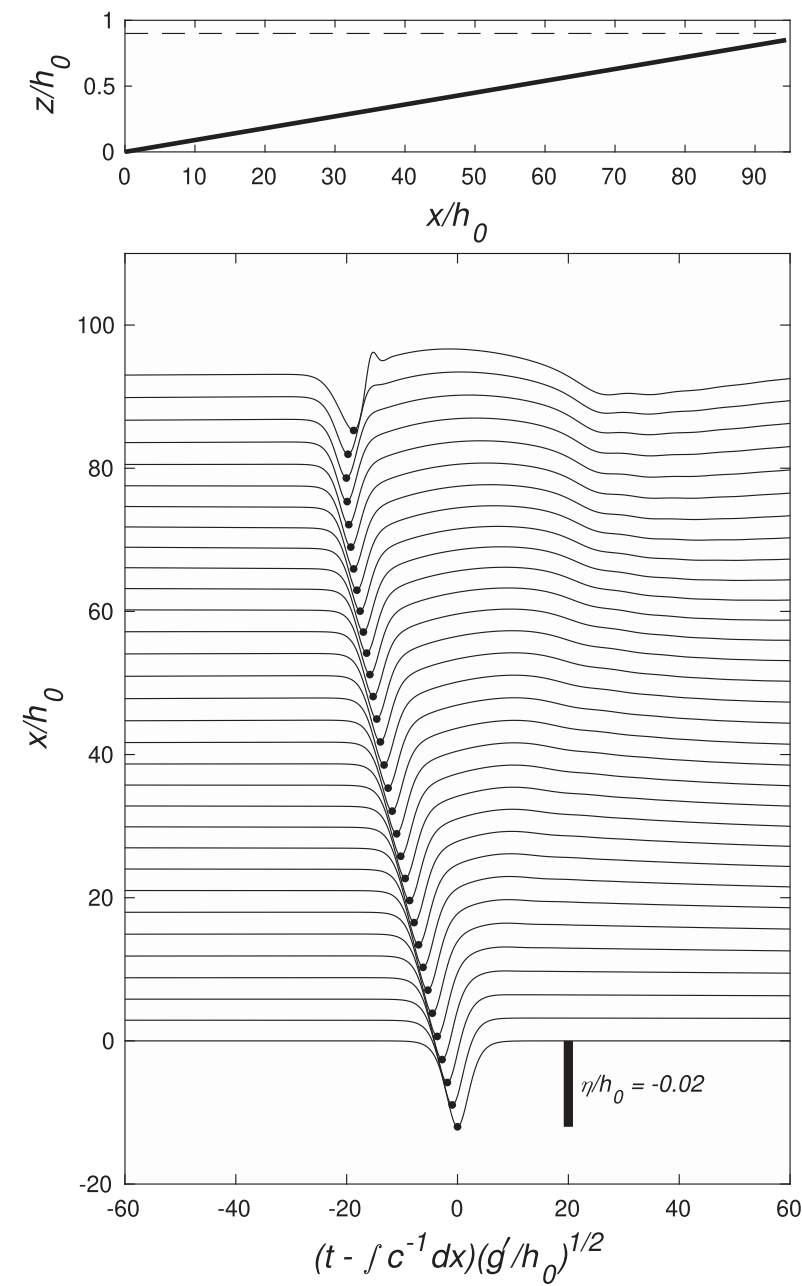

FIG. 4. As in Fig. 3, but for the rotating KdV solution with $L=44.5 \mathrm{~km}$.

is true for the $L=89 \mathrm{~km}$ case also shown in Fig. 2 by the diamonds.) An interesting feature of the $\mathrm{rKdV}$ solution in Fig. 5 is the evolution of a trailing solitary-like wave that emerges from steepening of the trailing inertiagravity wave radiation (see also Grimshaw et al. 1998a) from a localized zone where $3 \mu \gamma^{-1} \eta_{x x}>1$ (Grimshaw et al. 2012, 2014). As this trailing soliton propagates up the slope its evolves much as shown for the $L=44.5 \mathrm{~km}$ cases in Figs. 3 and 4, where the inhomogeneity effect is stronger. This is not surprising in view of the conditions once this soliton has emerged. Taking the wave starting near $x / h_{0}=225$, then the appropriate $s_{0} \approx 4$ and $L \approx 65$ $\mathrm{km}$. The wave amplitude is about the same as the initial wave. Together these give $G_{S} \approx 2$, so that the effect of radiation damping on this trailing solitary wave is relatively weak as shown in the figure. In this example the trailing radiation does not have enough time for an organized envelope soliton to emerge. However, a longer slope (smaller $G_{S}$ ) could lead to formation of such a 

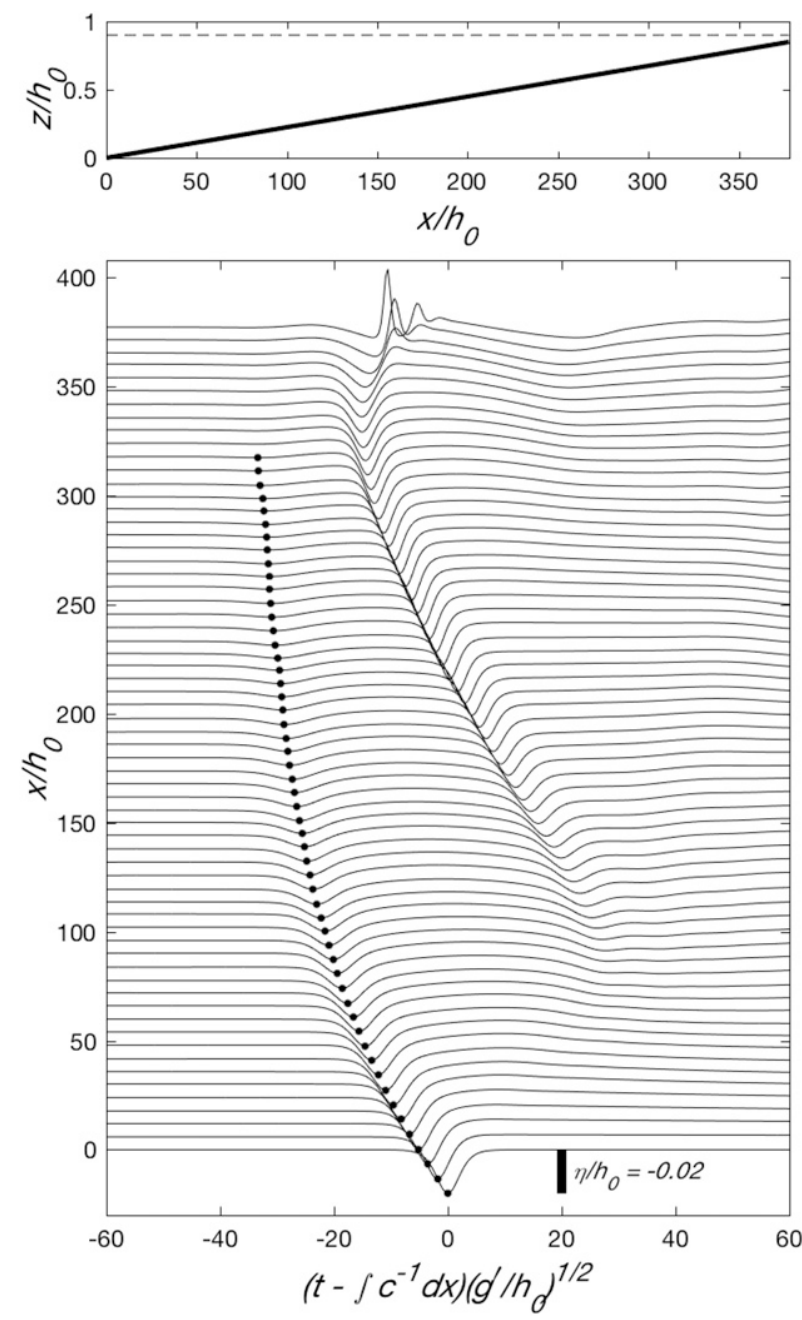

FIG. 5. As in Fig. 3, but for the rotating KdV solution with $L=178 \mathrm{~km}$.

wave packet. The subsequent evolution of the packet over the slope is of interest (cf. Yuan et al. 2018), but is beyond the scope of this paper.

As discussed above, the cubic nonlinear term $\alpha \eta^{2} \eta_{x}$ is frequently included in the brackets on the left-hand side of (1), where in the two-layer system (cf. Helfrich and Melville 2006)

$$
\alpha=-\frac{3 c}{8} \frac{\left(h_{1}^{2}+6 h_{1} h_{2}+h_{2}^{2}\right)}{\left(h_{1} h_{2}\right)^{2}},
$$

to give the rotating version of the Gardner equation. Two numerical runs, one nonrotating and the other rotating, have been made with the cubic term for $L=44.5 \mathrm{~km}$. The other parameters are as given above. The amplitudes of the solitary waves are shown in Fig. 2 by the filled symbols. Without rotation the cubic term leads to a modest amplitude reduction for $s \leqq 3$ compared to the

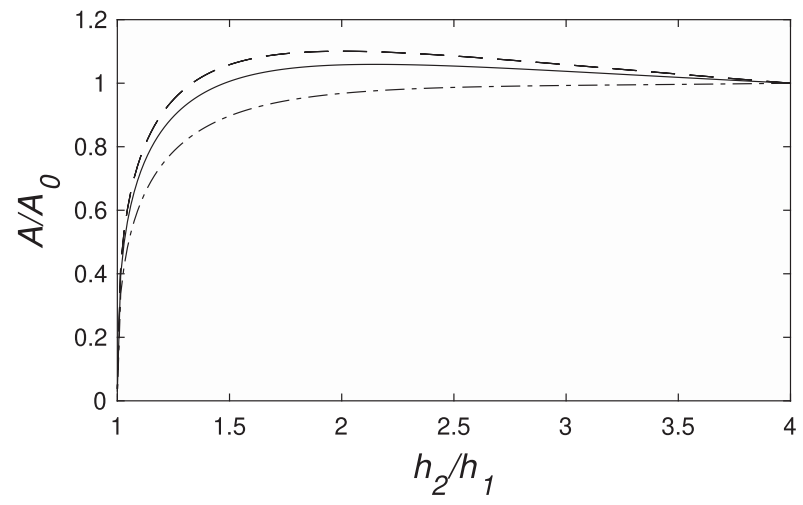

FIG. 6. Adiabatic theory for the South China Sea example with $h_{1}=100 \mathrm{~m}, h_{20}=400 \mathrm{~m}, \Delta \rho / \rho_{1}=2.5 \times 10^{-3}, f=5 \times 10^{-5} \mathrm{~s}^{-1}$, and $L=180 \mathrm{~km}$. The rotating examples with $A_{0}=-10 \mathrm{~m}$ (dash-dot) and $-100 \mathrm{~m}$ (solid) are shown along with the nonrotating solution (dashed line).

$\mathrm{rKdV}$ result, but the general behavior tracks the adiabatic theory. This is just where the cubic term is expected to be most significant. The reduction in soliton amplitude is expected since for the two-layer stratification $\alpha<0$ and solitons are limited to a maximum amplitude $A_{\max }=-\mu / \alpha$ (the flat-top, or conjugate state, wave), while there is no limit on KdV soliton amplitude (e.g., Grimshaw et al. 2004; Helfrich and Melville 2006). With strong rotation, the effect of the cubic term is even less significant since the inertia-gravity wave radiation has time to damp the soliton well before $s \rightarrow 1$ and $\mu \rightarrow 0$.

In the next example for shoaling of waves for $s_{0}>1$ we consider conditions roughly corresponding to those for South China Sea in Grimshaw et al. (2014). For estimates, we approximate the shelf-area bottom profile (the Northern onshore direction) shown in their Figs. 7 and 8 with a constant slope between $x=320$ and $500 \mathrm{~km}$. In this range $h_{1}=100 \mathrm{~m}, h_{20}=400 \mathrm{~m}$, and $L=180 \mathrm{~km}$ since $h_{1}=h_{2}$ occurs at $x \approx 500 \mathrm{~km}$. For $h_{1}$ we have taken an isopycnal level that lies slightly below the maximum of buoyancy frequency in their Fig. 2. We correspondingly take $\Delta \rho / \rho_{1}=2.5 \times 10^{-3}$. The latitude is about $20^{\circ}$, giving $f=5 \times 10^{-5} \mathrm{~s}^{-1}$. They take the initial solitary wave amplitudes to range from -60 to $-90 \mathrm{~m}$ at the deep basin location $x=54 \mathrm{~km}$. We are not modeling this region and so take $A_{0}=-100 \mathrm{~m}$ at $x=320 \mathrm{~km}$, our initial position. Actually this amplitude is well beyond the range of weakly nonlinear $\mathrm{KdV}$ model as $A_{0} / h_{1}=O(1)$, so we also consider $A_{0}=-10 \mathrm{~m}$ to illustrate the sensitivity to the amplitude. With these parameters $G_{S}=10.3$ and 32.7 for $A_{0}=-10$ and $-100 \mathrm{~m}$, respectively. Thus, in both cases the effect of inhomogeneity should dominate over rotational damping. This is illustrated in Fig. 6 where the adiabatic response $A / A_{0}$ is shown versus $s$ for these two initial amplitudes. For both the response is 
close to the nonrotating solution (dashed line) with the smaller wave only slightly more affected by rotation as anticipated from $G_{S} \sim A_{0}^{1 / 2}$. This is consistent with Figs. 4 and 5 in Grimshaw et al. (2014), in which rotating and nonrotating Gardner equation solutions show little effect of rotation on the evolution of the lead soliton for $x \geqslant 320 \mathrm{~km}$.

\section{b. Offshore propagation for $s_{0}<1$}

To obtain a more complete picture of the adiabatic theory we next briefly discuss examples of the three additional situations beginning with offshore propagation for $s_{0}<1$ where a point of polarity reversal is encountered at $s=1$. Figure 7 shows the adiabatic theory results for $A_{0}=10 \mathrm{~m}, h_{1}=100 \mathrm{~m}, h_{20}=25 \mathrm{~m}, g^{\prime}=5 \times 10^{-3} \mathrm{~m} \mathrm{~s}^{-2}$, and $f=10^{-4} \mathrm{~s}^{-1}$. Slope lengths $L=50$ and $200 \mathrm{~km}$ give $G_{S}=3.8$ and 0.95 , respectively. Without rotation the increasing depth leads to a monotonic decay of wave amplitude with complete extinction at $s=1$. The added decay induced by rotation gives complete extinction before $s=1$ for both slope lengths despite the relatively large value of $G_{S}$ for $L=50 \mathrm{~km}$. The symbols show results from numerical solutions to the $\mathrm{rKdV}$ equation. For $L=50 \mathrm{~km}$ the adiabatic theory predicts more rapid wave amplitude decay than the direct computation, although the trends do agree. For $L=200 \mathrm{~km}$ the theory and computation agree well in the initial stages of amplitude decay and diverge for $s \gtrsim 0.4$. As illustrated earlier, effects not accounted for in the adiabatic model lead to significant differences between theory and numerical solutions as $s \rightarrow 1$.

\section{c. Onshore propagation for $s_{0}<1$}

Figure 8 shows an example for $s_{0}<1$ of solitary wave propagation toward an internal beach, $s \rightarrow 0$, for $A_{0}=10 \mathrm{~m}, h_{1}=150 \mathrm{~m}, h_{20}=50 \mathrm{~m}, g^{\prime}=5 \times 10^{-3} \mathrm{~m} \mathrm{~s}^{-2}$, and $f=10^{-4} \mathrm{~s}^{-1}$. Again both $L=50 \mathrm{~km}$ and $200 \mathrm{~km}$ are considered. The nonrotating theory shows large amplitude growth well before the $s=0$ point indicating wave breaking or instability is to be expected. For $L=50 \mathrm{~km}$ rotation slows the initial amplitude growth, but ultimately the amplification due to the inhomogeneity dominates and ultimately exceeds the range of the underlying weakly nonlinear $\mathrm{KdV}$ model. The longer $L=200 \mathrm{~km}$ slope does lead to amplitude decay, although it is not complete by the time the beach is reached so that the predicted amplitude begins to grow. Results from numerical solutions to the $\mathrm{rKdV}$ model are also shown and, for these parameters, they agree very well with the adiabatic theory. However, the calculations are only shown for $s \gtrsim 0.1$ since the wave amplitudes for $L=50 \mathrm{~km}$ with rotation and the nonrotating example $(L=200 \mathrm{~km})$ become unrealistically large. The $L=200 \mathrm{~km}$ case with

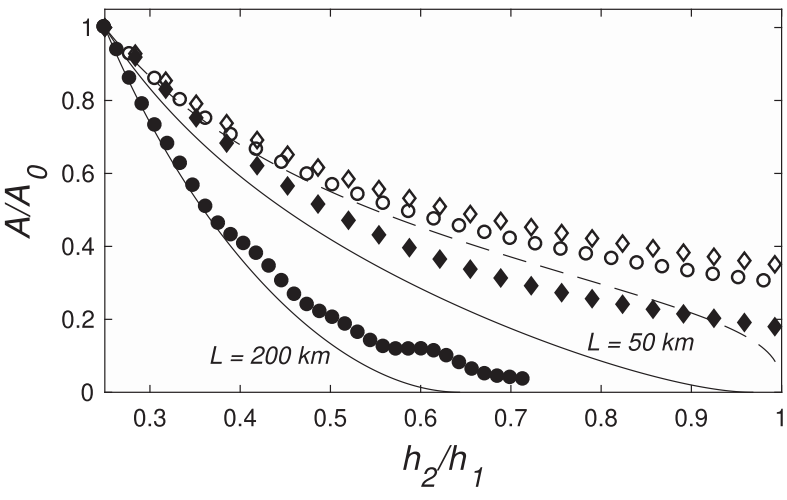

FIG. 7. Adiabatic theory vs $\mathrm{KdV}$ and $\mathrm{rKdV}$ numerical results for offshore propagation with $s_{0}=0.25 ; A_{0}=10 \mathrm{~m}, h_{1}=100 \mathrm{~m}, h_{20}=25 \mathrm{~m}$, $g^{\prime}=5 \times 10^{-3} \mathrm{~m} \mathrm{~s}^{-2}$, and $f=10^{-4} \mathrm{~s}^{-1}$. Slope lengths are $L=50$ and $200 \mathrm{~km}$. The dashed line is for the nonrotating case (valid for both $L$ values), and the solid lines are for the rotating cases with $L$ as indicated. The open (filled) symbols are for the respective $\mathrm{KdV}(\mathrm{rKdV})$ numerical solutions with diamonds for $L=50 \mathrm{~km}$ and circles for $L=200 \mathrm{~km}$.

rotation is more interesting as shown in Fig. 9. As the initial solitary wave decays due to the radiation damping the trailing radiation continues to propagate into shallower wave where it steepens to form several new solitary waves that amplify as they move onshore. For these trailing solitary waves the local lower-layer depth is shallower and there is less distance over which radiation damping can act, therefore the inhomogeneity dominates. This is similar to the evolution shown in Fig. 5 where the trailing radiation also evolves independently as it approaches the point of polarity reversal.

\section{d. Offshore propagation for $s_{0}>1$}

The last example shown in Fig. 10 involves offshore propagation for $s_{0}>1$. In this example the parameters used are $A_{0}=-10 \mathrm{~m}, h_{1}=25 \mathrm{~m}, h_{20}=100 \mathrm{~m}, g^{\prime}=5 \times$ $10^{-3} \mathrm{~m} \mathrm{~s}^{-2}$, and $f=10^{-4} \mathrm{~s}^{-1}$. Here the length scales $L=50$ and $200 \mathrm{~km}$ correspond to the distance from $x=0$ to where $h_{2}(L)=2 h_{20}$. As discussed above solitary wave amplitude is expected to decay due to both topographic inhomogeneity and rotation. Furthermore, since no singularity, that is, a point of polarity reversal or an internal beach, is encountered the initial solitary wave is expected to evolve with minimal departure from the assumed form (3) at all locations. This is indeed what is found in the full $\mathrm{rKdV}$ numerical solutions which, as shown Fig. 10, agree very well with the adiabatic theory.

\section{Concluding remarks}

This paper is intended to analyze the behavior of slowly varying solitary waves in the rKdV (Ostrovsky) equation when both factors, the Coriolis effect and the 


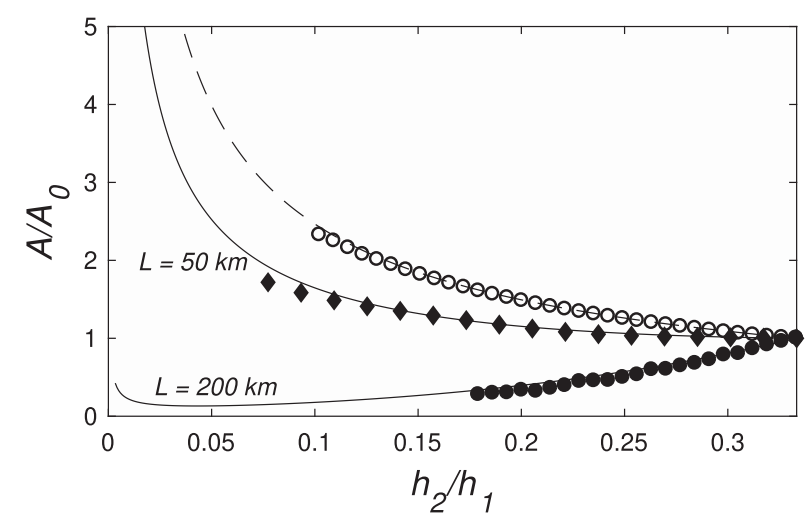

FIG. 8. Adiabatic theory vs $\mathrm{KdV}$ and $\mathrm{rKdV}$ numerical results for onshore propagation with $s_{0}=1 / 3 ; A_{0}=10 \mathrm{~m}, h_{1}=150 \mathrm{~m}, h_{20}=50 \mathrm{~m}$, $g^{\prime}=5 \times 10^{-3} \mathrm{~m} \mathrm{~s}^{-2}$, and $f=10^{-4} \mathrm{~s}^{-1}$. Slope lengths are $L=50$ and $200 \mathrm{~km}$. The dashed line is for the nonrotating case (valid for both $L$ values), and the solid lines are for the rotating cases with $L$ as indicated. The open (filled) symbols are for the respective $\mathrm{KdV}(\mathrm{rKdV})$ numerical solutions with diamonds for $L=50 \mathrm{~km}$ and circles for $L=200 \mathrm{~km}$.

horizontal inhomogeneity, compete. Based on the general adiabatic solution obtained in Grimshaw et al. (2014), we demonstrated possible scenarios of soliton evolution and death in a two-layer ocean, and specifically, in that with the constant bottom slope. In the case of a negative polarity solitary wave for $s_{0}>1$ the onshore propagating wave never reaches the beach as a unique entity. Two possible scenarios of its destruction are 1) reaching the point of equal layer thicknesses where the coefficient of the quadratic nonlinearity $\mu$ goes to zero, and 2) terminal damping due to inertiagravity wave radiation at a shorter distance, when the lower layer is still thicker than the upper layer. We considered examples of both these scenarios with the "competition" parameter $G_{S}$ selecting between them. Along with constructing the adiabatic solution, direct numerical calculations of the $\mathrm{rKdV}$ equation were obtained and compared with the adiabatic theory in several realistic cases, including the case roughly corresponding to the shelf conditions in South China Sea described in Grimshaw et al. (2014). The agreement was quite good and gives support to the adiabatic theory and the description of the competition process through $G_{S}$. While we have concentrated on the simple two-layer stratification and a constant slope for both analytical progress and illustrative reasons, the qualitative behavior found here will persist in general continuous stratifications and nonconstant slopes.

We have also briefly considered three other possible scenarios. When $s_{0}<1$, a solitary wave of elevation propagating offshore will also encounter a point of polarity reversal. Again competition between topographic
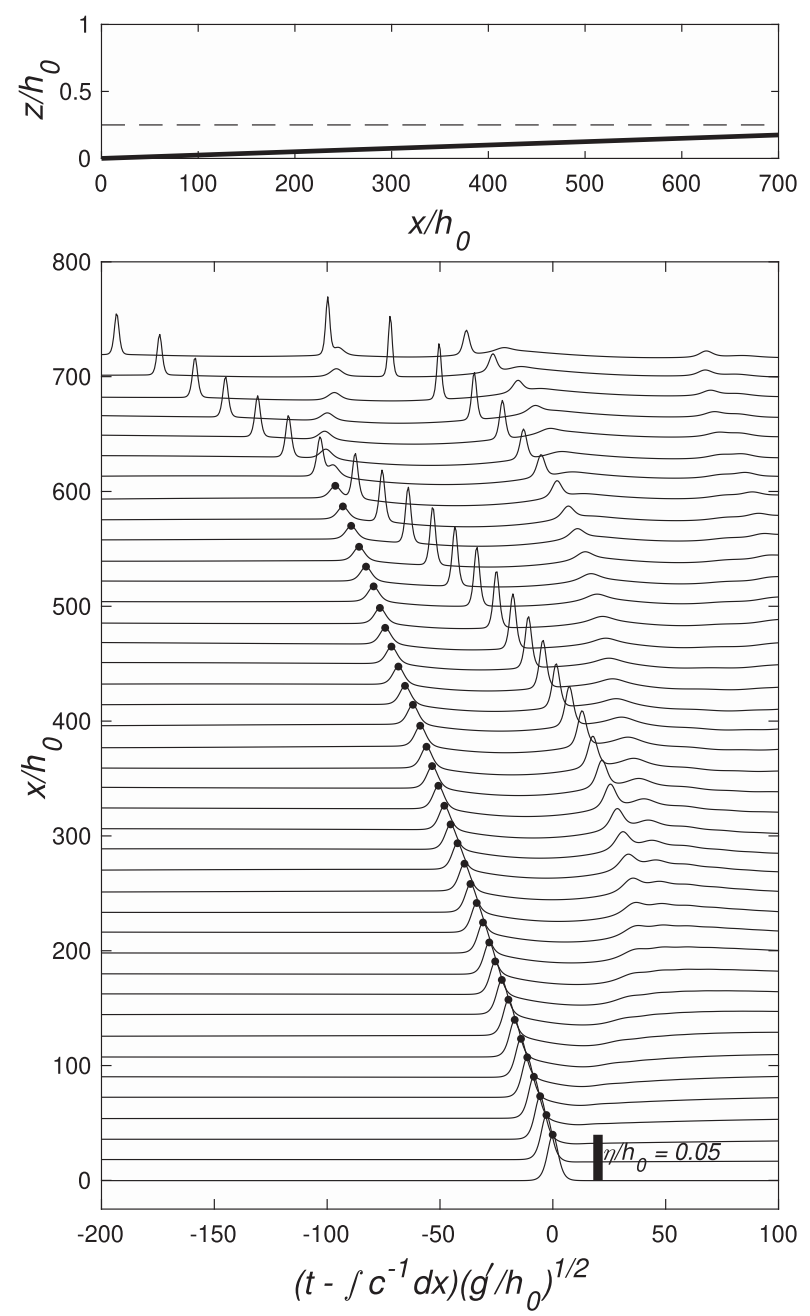

FIG. 9. The rKdV solution for the parameters of Fig. 8 and $L=200 \mathrm{~km}$.

inhomogeneity and radiation damping will occur and wave extinction due to these two effects can be analyzed similarly. The case of a wave of elevation for $s_{0}<1$ approaching an internal beach was also discussed. In that situation the singularity at the beach leads to unbounded amplitude growth as $s \rightarrow 0$ that can be quenched by rotational effects. However, the trailing radiation propagating onshore is itself subject to effects of the singularity. Last, the relatively simple situation of a wave of depression, $s_{0}>1$, propagating offshore, where no singularity is encountered, was discussed. While the exploration of parameter values was far from complete, the values chosen are representative of the coastal ocean and the results are indicative of the possible wave evolution scenarios. Taken together, we hope that these results illustrate the value and stimulate a wider use of asymptotic theory for solitary waves in rotating ocean. 


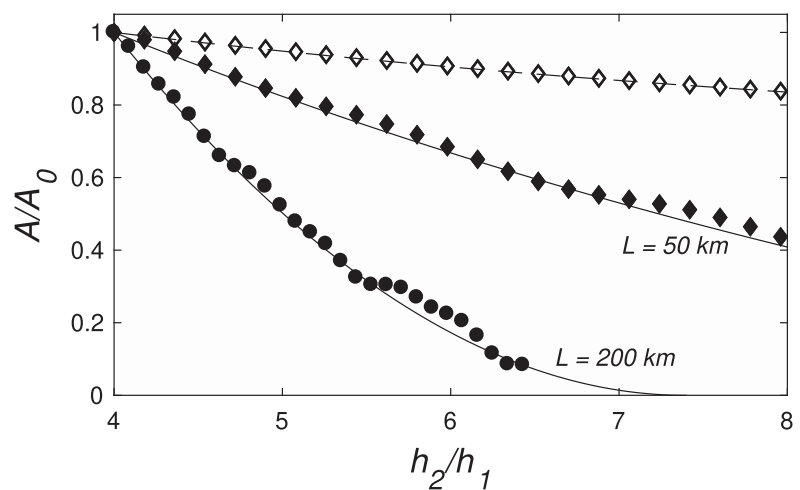

FIG. 10. Adiabatic theory vs $\mathrm{KdV}$ and $\mathrm{rKdV}$ numerical results for offshore propagation with $s_{0}=4 ; A_{0}=-10 \mathrm{~m}, h_{1}=25 \mathrm{~m}, h_{20}=100 \mathrm{~m}$, $g^{\prime}=5 \times 10^{-3} \mathrm{~m} \mathrm{~s}^{-2}$, and $f=10^{-4} \mathrm{~s}^{-1}$. Slope lengths (see text) are $L=50$ and $200 \mathrm{~km}$. The dashed line is for the nonrotating case (valid for both $L$ values), and the solid lines are for the rotating cases with $L$ as indicated. The open (filled) symbols are for the respective $\mathrm{KdV}(\mathrm{rKdV})$ numerical solutions with diamonds for $L=50 \mathrm{~km}$ and circles for $L=200 \mathrm{~km}$.

As this paper was under review, the paper by Stepanyants (2019) was published which uses a similar adiabatic theory approach to explore surface and internal solitary wave evolution with the primary goal of determining specific depth profile that leads to constant displacement amplitude (but not wavelength or energy of the solitary wave) where the decay by radiation is precisely countered by the inhomogeneous effects. An example of an adiabatic solution for the shoaling of an internal solitary wave of depression with rotation is presented. However, companion numerical comparisons are not given. Furthermore, there is no discussion of the other possible scenarios nor is there an exploration of the limitations of the adiabatic approach as is presented here.

Here we have mostly limited ourselves to the dynamics of a soliton as a distinguished entity to the initial stage of soliton destruction. Further evolution of the internal soliton is known to be complicated. The behavior of radiation in a homogeneous medium with rotation was considered by Grimshaw et al. (1998a) and its long-time evolution by Grimshaw and Helfrich (2008) and Whitfield and Johnson (2014) who have shown that a sufficiently long-time evolution of the soliton remnants can form a nonlinear wave packet which can be considered as an envelope soliton propagating at near the maximal group velocity of the linearized version of (1) and satisfying a nonlinear Shrödinger equation. Grimshaw et al. (2017) found observational evidence for these wave packets, so that the topic of their evolution over variable topography is certainly relevant. Yuan et al. (2018) considered the closely related issue of the evolution of undular bores, but not envelope solitons, over variable topography which also pointed to the rich behavior of nonlinear internal waves under the influence of variable topography and rotation. On the other hand, in either the nonrotating case, or that with weak rotational damping effects, after reaching the location where $h_{1}=h_{2}(\mu=0$ for a general continuous stratification) a scattered wave packet will develop from which one or more solitons of opposite polarity can be formed. The description of this stage typically requires the inclusion of the cubic nonlinearity, that is, the rotating Gardner equation with variable coefficients. Additionally, in certain parameter regimes the cubic nonlinearity could be significant prior to the scattering region as Obregon et al. (2018) have recently shown in a study of the adiabatic decay of solitons in the constant-coefficient, rotating Gardner equation. This adiabatic theory could be extended to include inhomogeneity through variable topography. Last, energy losses through either bottom boundary drag or internal instabilities will slowly extract energy. Parameterizations of these processes can be included in either the rKdV or rotating-Gardner model, and their competition with the effects of inhomogeneities and rotational radiation decay could be explored. These problems will be the subjects of future investigation.

Acknowledgments. LAO is thankful to Yu. Stepanyants for broad discussions of mutual benefit. KRH was supported by Grant N00014-18-1-2542 from the Office of Naval Research.

\section{APPENDIX}

\section{Two-Layer $Q(x)$}

In the absence of background shear current, the modal function $\phi(z)$ satisfies the known boundary value problem for a Boussinesq fluid:

$c^{2} \frac{d^{2} \phi}{d z^{2}}+N^{2}(z) \phi=0, \quad \phi(-h)=\phi(0)=0$.

Here $N=\left(-g / \rho_{0} d \rho / d z\right)^{1 / 2}$ is the Brunt-Väisälä frequency. Multiplying (A1) by $\phi$ and integrating over $z$, we have

$$
c^{2}=\frac{\int_{-h}^{0} N^{2} \phi^{2} d z}{\int_{-h}^{0}(d \phi / d z)^{2} d z} .
$$

In a two-layer model, $N^{2}=\left(g \Delta \rho / \rho_{0}\right) \delta\left(z-z_{i}\right)$, where $z_{i}$ is the interface level and $\Delta \rho$ is the density jump. The numerator in (A2) is $g \Delta \rho / \rho_{0}=g^{\prime}$, and if $h$, and possibly $z_{i}$, 
vary in $x$, but the density jump is fixed, the numerator is independent of $x$. As a result,

$$
c^{2} \int_{-h}^{0}\left(\frac{d \phi}{d z}\right)^{2} d z=\text { const }
$$

and comparison with (2) shows that $Q(x) \propto c(x)$. As mentioned, a constant factor in $Q$ does not affect the corresponding term in (1). Thus, in the two-layer case $Q(x)$ can be replaced by $c(x)$. The version of (1) using $Q=c$ was recently used by Stepanyants (2019) without proof.

\section{REFERENCES}

Farmer, D., Q. Li, and J.-H. Park, 2009: Internal wave observations in the South China Sea: The role of rotation and nonlinearity. Atmos.-Ocean, 47, 267-280, https://doi.org/10.3137/OC313.2009.

Grimshaw, R., and K. Helfrich, 2008: Long-time solutions of the Ostrovsky equation. Stud. Appl. Math., 121, 71-88, https:// doi.org/10.1111/j.1467-9590.2008.00412.x.

__ , J.-M. He, and L. Ostrovsky, 1998a: Terminal damping of a solitary wave due to radiation in rotational systems. Stud. Appl. Math., 101, 197-210, https://doi.org/10.1111/ 1467-9590.00090.

— E. Pelinivsky, and T. Talipova, 1998b: Solitary wave transformation due to a change in polarity. Stud. Appl. Math., 101, 357-388, https://doi.org/10.1111/1467-9590.00098.

,,--- , and A. Kurkin, 2004: Simulation of the transformation of internal solitary waves on oceanic shelves. J. Phys. Oceanogr., 34, 2774-2791, https://doi.org/10.1175/ JPO2652.1.

_- K. Helfrich, and E. R. Johnson, 2012: The reduced Ostrovsky equation: Integrability and breaking. Stud. Appl. Math., 129, 414-436, https://doi.org/10.1111/j.1467-9590.2012.00560.x.

C. Guo, K. Helfrich, and V. Vlasenko, 2014: Combined effect of rotation and topography on shoaling oceanic internal solitary waves. J. Phys. Oceanogr., 44, 1116-1132, https:// doi.org/10.1175/JPO-D-13-0194.1.

_ _ J. da Silva, and J. Magalhaes, 2017: Modeling and observations of oceanic nonlinear internal wave packets affected by the Earth's rotation. Ocean Modell., 116, 146-158, https:// doi.org/10.1016/j.ocemod.2017.06.009.

Helfrich, K. R., 2007: Decay and return of internal solitary waves with rotation. Phys. Fluids, 19, 026601, https://doi.org/10.1063/ 1.2472509 .

—, and W. K. Melville, 2006: Long nonlinear internal waves. Annu. Rev. Fluid Mech., 38, 395-425, https://doi.org/10.1146/ annurev.fluid.38.050304.092129.

_ —_ , and J. W. Miles, 1984: On interfacial solitary waves over slowly varying topography. J. Fluid Mech., 149, 305-317, https://doi.org/10.1017/S0022112084002664.

Obregon, M., N. Raj, and Y. Stepanyants, 2018: Adiabatic decay of internal solitons due to Earth's rotation within the framework of the Gardner-Ostrovsky equation. Chaos, 28, 033106, https:// doi.org/10.1063/1.5021864.

Ostrovsky, L., 1978: Nonlinear internal waves in a rotating ocean. Oceanology, 18, 119-125.

Pelinovsky, E. N., M. A. Rauevsky, and S. Shavratsky, 1977: The Korteweg-de Vries equation for nonstationary internal waves in an inhomogeneous ocean. Izv., Atmos. Ocean. Phys., 13, 226-228.

Stepanyants, Y., 2019: The effects of interplay between the rotation and shoaling for a solitary wave on variable topography. Stud. Appl. Math., 142, 465-486, https://doi.org/10.1111/sapm.12255.

Talipova, T. G., O. E. Kurkina, E. A. Rouvinskaya, and E. N. Pelinovsky, 2015: Propagation of solitary internal waves in two-layer ocean of variable depth. Izv., Atmos. Ocean. Phys., 51, 89-97, https://doi.org/10.1134/S0001433815010107.

Whitfield, A. J., and E. R. Johnson, 2014: Rotation-induced nonlinear wavepackets in internal waves. Phys. Fluids, 26, 056606, https://doi.org/10.1063/1.4879075.

Yuan, C., R. Grimshaw, and E. Johnson, 2018: The evolution of internal undular bores over a slope in the presence of rotation. Stud. Appl. Math., 140, 465-482, https://doi.org/10.1111/ sapm.12207. 